\title{
An impulsive bathtub vortex
}

\author{
Peder A. Tyvand and Kjetil B. Haugen \\ Agricultural University of Norway \\ Department of Mathematical Sciences and Technology \\ P.O. Box 5003 \\ $5432 \AA ̊ s$ \\ Norway
}

\section{Introduction}

The bathtub vortex is a famous phenomenon. The experiments by Shapiro (1962) and Trefethen et al. (1965) indicate that the circulation of a bathtub vortex may possibly be governed by the rotation of the Earth. This is only true if the tank is wide enough, the draining is slow enough and the water in the tank has settled for many hours. An attempt at explaining theoretically the influence of the Earth's rotation was presented by Marris (1967). Here the free surface was assumed flat, and a simplified flow field was considered. This lead to a somewhat unrealistic exponential growth of the vorticity. Lord Kelvin's circulation theorem for axisymmetric inviscid flow in an inertial system gives $(d / d t)\left(c d^{2}\right)=0$. Here $t$ is time, $c$ is the vorticity and $d$ is the diameter of a material circle of fluid particles. This formula can be applied directly to Shapiro's experiments. It gives a reasonable estimate of $1 \mathrm{~cm}$ for the diameter of the circular particle paths near the sink, provided the flow is due to the rotation of the Earth. Lundgren (1985) presented a consistent model where the free-surface deformation was taken into account. He considered fluid in a container with rapid rotation (small Rossby number). Our present theoretical approach corresponds to the inviscid model of Lundgren (1985), but covers a different parameter regime: We assume a rapid impulsive start of the bottom drain, instead of the gradual flow development assumed by Lundgren. Moreover, we assume a slow container rotation (high Rossby number), so that our theory can be compared with a bathtub rotating with the rotating Earth. We will show that the vorticity amplification in an impulsive bathtub vortex is far too small to have anything to do with the rotation of the Earth. 


\section{Analysis}

We consider inviscid flow of a homogeneous and incompressible fluid of density $\rho$. The fluid is confined in a wide container. The gravitational acceleration is $g$. At time $t<0$ there is a steady rotation with angular velocity $\Omega$. The origin is placed in the surface midpoint. We introduce cylindrical coordinates $(r, \theta, z)$, with the associated velocity components $\left(v_{r}, v_{\theta}, w\right)$, respectively. The bottom of the container is given by $z=-D$. We are going to study the swirling dip formation due to a point sink impulsively started in the midpoint of the container bottom. The impulsive point sink has a constant volume flux $Q$. We define the Froude number $F$ and the Rossby number $\epsilon^{-1}$, respectively, as follows

$$
F=\frac{Q}{4 \pi \sqrt{g D^{5}}}, \quad \epsilon=\frac{\Omega D^{3}}{Q} .
$$

The units of dimensionless length, time, velocity and pressure are $D, D^{3} / Q$, $Q / D^{2}$ and $\rho g D$, respectively. The dimensionless components of the Euler equation of motion in the radial and azimuthal directions are (Lundgren 1985):

$$
\begin{gathered}
\frac{\partial v_{r}}{\partial t}+v_{r} \frac{\partial v_{r}}{\partial r}+w \frac{\partial v_{r}}{\partial z}-\frac{v_{\theta}^{2}}{r}=-\frac{1}{16 \pi^{2} F^{2}} \frac{\partial p}{\partial r} \\
\frac{\partial v_{\theta}}{\partial t}+v_{r} \frac{\partial v_{\theta}}{\partial r}+w \frac{\partial v_{\theta}}{\partial z}+\frac{v_{r} v_{\theta}}{r}=0 .
\end{gathered}
$$

The remaining governing equations are the same as in the corresponding irrotational problem (Haugen and Tyvand 2003). The flow field is described in terms of a small-time expansion:

$$
\mathbf{v}=\epsilon r \mathbf{i}_{\theta}+H(t)\left(\mathbf{v}_{0}+t \mathbf{v}_{1}+t^{2} \mathbf{v}_{2}+\ldots\right) .
$$

The first term is the steady rigid-body rotation. $H(t)$ is the Heaviside unit step function. We will assume a flat initial surface, which implies the condition $\epsilon \ll 0.03 / F$. The flow during dip formation is consistently split into a potential flow (Haugen and Tyvand 2003) and an azimuthal flow expressed as

$$
v_{\theta}(r, z, t)=\epsilon r+H(t)\left(t v_{1 \theta}+t^{2} v_{2 \theta}+t^{3} v_{3 \theta}+\ldots\right) .
$$

This azimuthal flow at the surface will be evaluated to third order:

$$
\left.v_{\theta}\right|_{z=\eta}=\epsilon r+H(t)\left(t v_{1 \theta}+t \frac{\partial v_{1 \theta}}{\partial z} \eta+\frac{t}{2} \frac{\partial^{2} v_{1 \theta}}{\partial z^{2}} \eta^{2}+t^{2} v_{2 \theta}+t^{2} \frac{\partial v_{2 \theta}}{\partial z} \eta+t^{3} v_{3 \theta}\right)_{z=0} .
$$


This azimuthal surface flow grows rapidly, as a cubic function of time

$$
\left.v_{\theta}\right|_{z=\eta}=\epsilon r+H(t) \frac{t^{3} \epsilon}{3}\left(\frac{1}{16 \pi^{2} F^{2}}+2 \eta_{2}\right) \frac{d \eta_{1}}{d r}+O\left(t^{4}\right)
$$

This gives the vertical component of the surface vorticity

$\left.c\right|_{z=\eta}=2 \epsilon+H(t) \frac{t^{3} \epsilon}{3}\left(\left(\frac{1}{16 \pi^{2} F^{2}}+2 \eta_{2}\right)\left(\frac{d^{2} \eta_{1}}{d r^{2}}+\frac{1}{r} \frac{d \eta_{1}}{d r}\right)+2 \frac{d \eta_{1}}{d r} \frac{d \eta_{2}}{d r}\right)+O\left(t^{4}\right)$.

This vorticity is evaluated at the central surface point above the sink

$$
\left.c\right|_{(r, z)=(0, \eta)}=\epsilon\left(2+H(t) t^{3}\left(0.00398687 F^{-2}-0.0807627\right)\right) .
$$

Let us now neglect the rotational influence on the surface dip (assuming $\epsilon$ small). Then we can apply the result from (Haugen and Tyvand 2003, eq. 73) valid at the threshold of dip formation: $F=0.10944$. This critical dip reaches the bottom sink at dimensionless time $t=t_{c}=3.67$. Just at this moment when the surface is being swallowed by the sink, we have

$$
\left.c\right|_{(r, z)=(0,-1)}=2 \epsilon(1+6.2312)
$$

where $\eta\left(0, t_{c}\right)=-1$ by definition. The first term in the parenthesis of eq. (10) represents rigid-body rotation. The rigid-body vorticity is thus magnified by a factor 7.2312 as the critical rotating dip reaches the bottom sink. We will later modify this result for the surface vorticity by taking into account the effect of rotation on the critical Froude number. Note that the present theory does not say anything about the flow after the free-surface dip has been swallowed into the sink. The dominating pressure deviation at the free surface can be integrated up to give

$$
\left.p\right|_{z=\eta}=H(t) \epsilon^{2}\left(\frac{2}{3} \eta_{1} t^{3}+O\left(t^{4}\right)\right) .
$$

We can now extend the formula given by Haugen and Tyvand (2003, eq. 38) for the total third-order elevation, to account for rotation

$$
\eta_{3}=\eta_{3 S}+\eta_{3 G}+\eta_{3 C}+\frac{2}{3} \epsilon^{2} \eta_{1}
$$

Thereby we can modify the result from Haugen and Tyvand (2003, eq. 73) for the total third-order elevation above the impulsive bottom sink

$$
\eta(0, t)=-\frac{G}{\pi} t-0.06414 t^{2}+\left(\frac{0.00061544}{F^{2}}-0.0324923-\frac{2 G \epsilon^{2}}{3 \pi}\right) t^{3}+O\left(t^{4}\right) .
$$

Here $G=0.915965594$.. is Catalan's constant. 


\section{Concluding remarks}

We have shown that the bathtub vortex starting impulsively leads to an amplification of the initial vorticity by a factor of seven compared with a weak initial rigid-body rotation (large Rossby number). This demonstrates that an impulsive bathtub vortex can never be caused by the Earth's rotation, as this would require an amplification of order $10^{4}$ or more (Shapiro 1962). An impulsive flow has too strong vertical component and cannot shrink the material circles sufficiently on their way into the sink during dip formation. The present work is an extension of the model by Haugen and Tyvand (2003), to include rotational azimuthal flow. Our previous paper investigated the nonlinear time-dependent free-surface flow due to an impulsive point sink at the bottom of a fluid layer. The same problem in two dimensions had been investigated by Landrini and Tyvand (2001). Earlier papers by Tyvand (1992) and Miloh and Tyvand (1993) analyzed the time-dependent nonlinear free-surface flows due to a submerged impulsive line sink and point sink with infinite depth.

\section{References}

[1] K.B. Haugen and P.A. Tyvand (2003) Free-surface evolution due to an impulsive bottom sink at uniform depth. Phys. Fluids 15, 742-751.

[2] M. Landrini and P.A. Tyvand (2001) Generation of water waves and bores by impulsive bottom flux. J. Engng. Math. 39, 131-170.

[3] T.S. Lundgren (1985) The vortical flow above the drain-hole in a rotating vessel. J. Fluid Mech. 155, 381-412.

[4] A.W. Marris (1967) Theory of the bathtub vortex. Trans. ASME: J. Appl. Mech. 61, 11-15.

[5] T. Miloh and P.A. Tyvand (1993) Non-linear transient free-surface flow and dip formation due to a point sink. Phys. Fluids A5, 1368-1375.

[6] A. Shapiro (1962) Bath-tub vortex. Nature 196, 1080-1081.

[7] L.M. Trefethen, R.W. Bilger, P.T. Fink, R.E. Luxton and R.I. Tanner (1965) The bath-tub vortex in the Southern Hemisphere. Nature, 207, 1084-1085.

[8] P.A. Tyvand (1992) Unsteady free-surface flow due to a line source. Phys. Fluids A4, 671-676. 